Supplement of The Cryosphere, 14, 3407-3424, 2020

https://doi.org/10.5194/tc-14-3407-2020-supplement

(C) Author(s) 2020. This work is distributed under

the Creative Commons Attribution 4.0 License.

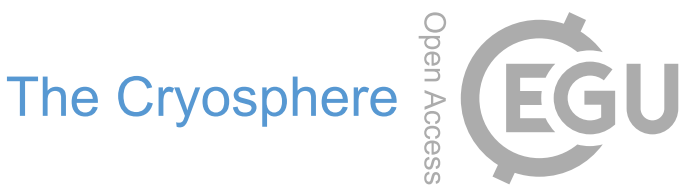

(c) (1)

Supplement of

\title{
Diagnosing the sensitivity of grounding-line flux to changes in sub-ice-shelf melting
}

\section{Tong Zhang et al.}

Correspondence to: Stephen F. Price (sprice@lanl.gov)

The copyright of individual parts of the supplement might differ from the CC BY 4.0 License. 


\section{S1 Correlations between $N_{r}$ and various metrics}

While the main goal of the study was to investigate the ability of the buttressing number $\left(N_{b}\right)$ to predict the GLF response number $\left(N_{r}\right)$, we also assessed the predictive skill of a range of other metrics. This was motivated by the lack of a clear theoretical justification for expecting $N_{b}$ to predict $N_{r}$ and a concern that observed correlations may be spurious. To this end,

5 we calculated correlation coefficients for a variety of locally defined metrics related to the stress state of the ice shelf (Table S1). Correlations for each metric were calculated for the MISMIP+ and Larsen C domains and in both cases, the calculations were made with and without filtering points by the shear metric, $m_{s}$ (Eq. 12).

The first set of metrics are the buttressing number $\left(N_{b}\right)$ defined for a number of different normal directions, as discussed in the main paper. We also considered normal directions rotated $30^{\circ}$ and $45^{\circ}$ from the $\mathbf{n}_{p 1}$ direction, which was motivated by the observation in Figs. 5a and S6, respectively, of maximum correlations in those directions. Additionally, we included the shear metric, $m_{s}$, and the quantity "backstress" (Thomas, 1979; Thomas and MacAyeal, 1982; MacAyeal, 1987), which as described in Appendix B is equivalent to $N_{b}\left(\mathbf{n}_{f}\right) N_{0}$. For completeness, we also assess the principal stresses, the maximum shear stress, and the stress in the flow direction. Finally, we include the equivalent ocean pressure, $N_{0}$ (which is proportional to ice thickness), and the two terms of the force balance used to calculate backstress.

There is not a single metric that has the highest correlation across all four cases. The metrics that perform the best across all cases are backstress $\left(B_{s}\right)$ and the buttressing number $\left(N_{b}\right)$ using normal directions between the $\mathbf{n}_{p 1}$ and $\mathbf{n}_{p 2}$ directions. In general, $N_{b}\left(\mathbf{n}_{p 1}\right)$ does not perform particularly well, reiterating the conclusions in the main paper that it cannot be recommended as a reliable predictor of changes in flux across the grounding line. Also of note are what appear to be spurious correlations. For example, the driving force, $F_{D}$, and the ocean backpressure, $N_{0}$, generate the largest correlation coefficients for the MISMIP+ case limited to points where $m_{s}<1$, but have no particular skill when all points are considered. Similarly, the longitudinal stretching force $\left(F_{L}\right)$ for the MISMIP+ with $m_{s}<1$ has high skill, but yields correlation coefficients close to 0 for the other cases. There is no theoretical reason to expect any of these metrics to relate directly to change in flux across the grounding line. These examples highlight the need for caution in extrapolating what appear to be strong correlations but are based on the analysis of a specific ice shelf configuration.

The conclusion of this detailed correlation analysis confirms that of the other analyses in the paper: no single metric can be identified that can be used with confidence to predict $N_{r}$ in general. While high correlations can be found in specific circumstances, there is not a single metric with universal skill in predictability, and applications to the real-world ice shelf fail to provide any relationships with practical utility. 


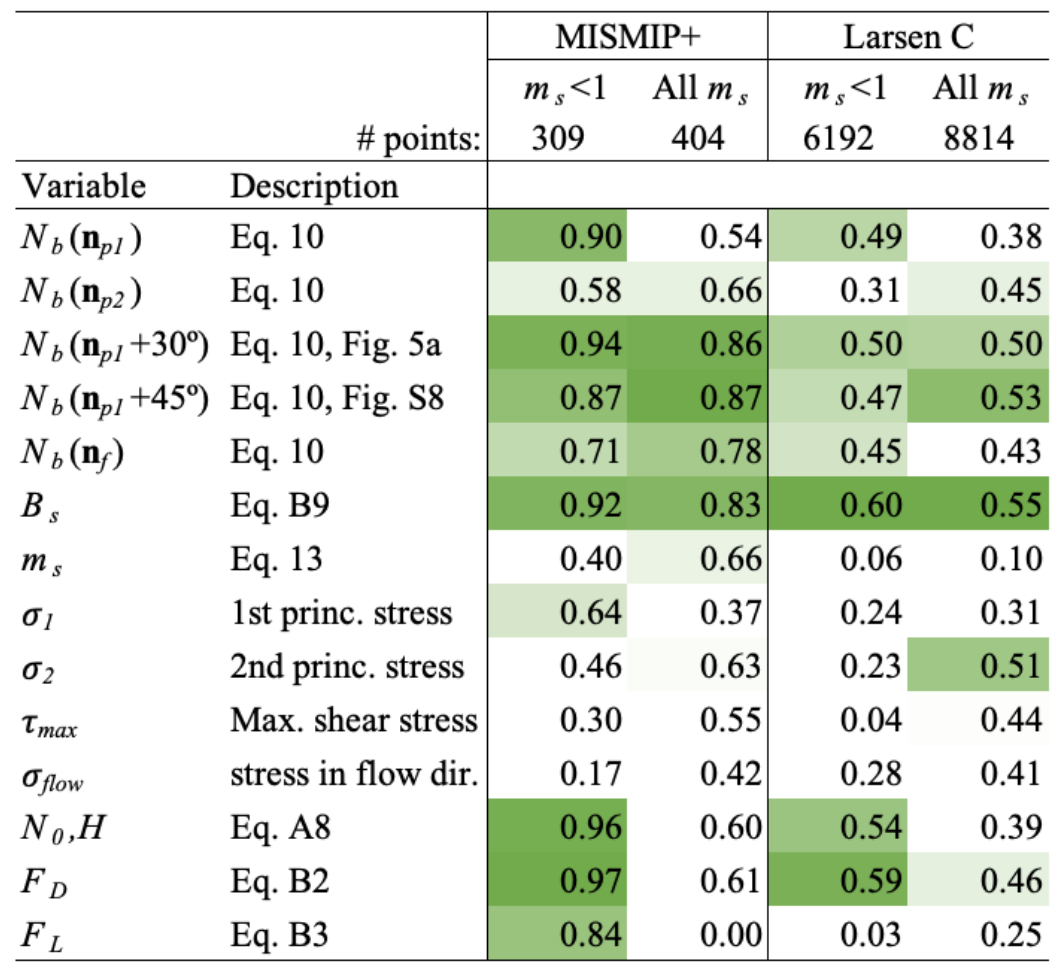

Table S1. Correlation coefficients $(r)$ between various metrics and $N_{r p}$ calculated for the MISMIP+ and Larsen C model domains. For each domain, $r$ values are reported for two situations: 1) where the shear metric $m_{s}$ is less than 1 , and 2) for all values of $m_{s}$. In both situations, grid cells intersecting the grounding line and grid cells adjacent to those points are excluded. Also, for both situations applied to the MISMIP+ domain, points on the ice shelf east of $x=480 \mathrm{~km}$ are excluded. Values lower than the 50th percentile are shown with a white background, and values between the 50th and 99th percentile are shown with increasingly dark green shading. 
a

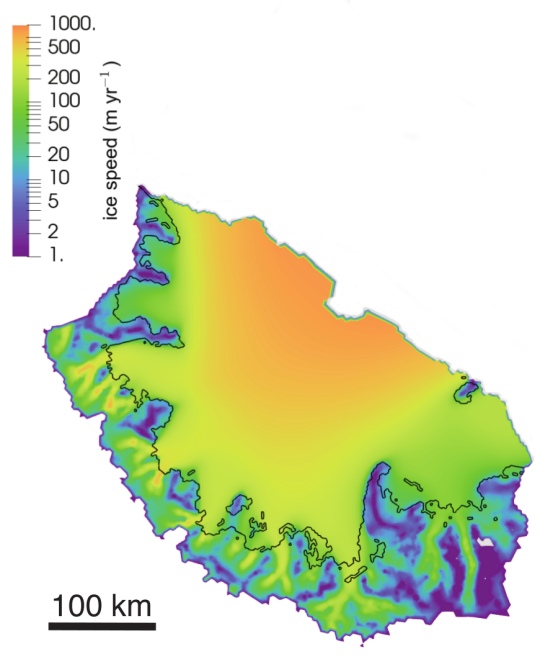

b

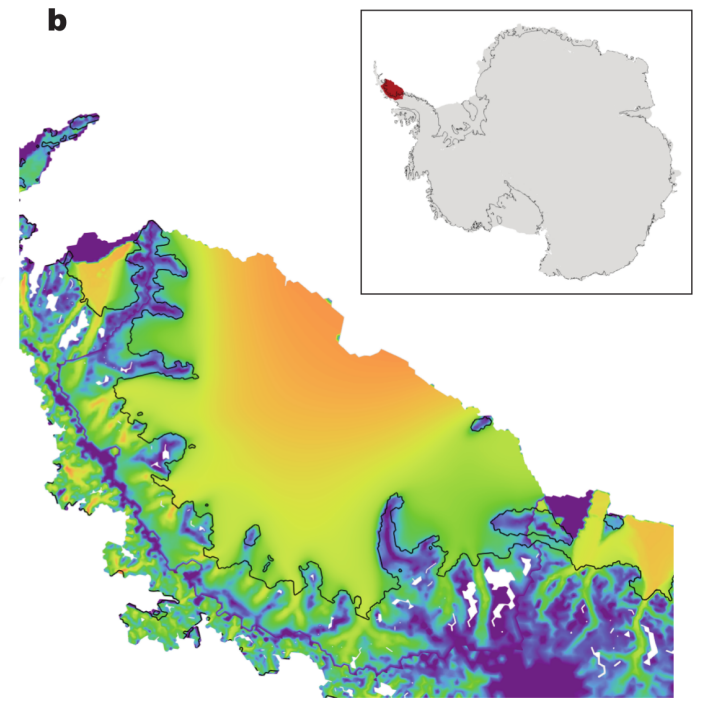

Figure S1. The modeled (a) and observed (b) surface ice speed for Larsen C Ice Shelf. Note that after 100 yr relaxation, the grounding line position in the modeled result is different from present-day observation.
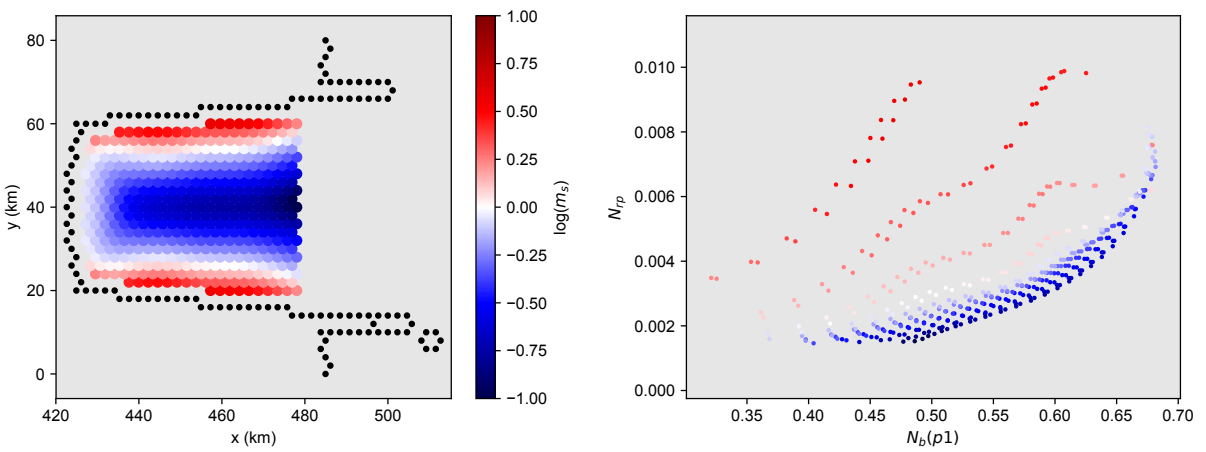

Figure S2. Shear ratio metric, $m_{s}$. a) Map of $\log \left(m_{s}\right)$. Positive values correspond to $m_{s}>1$ where the maximum shear stress is large relative to the average normal stress, while negative values correspond to $m_{s}<1$. Black dots indicate grounding line location. b) Plot of $N_{b}(p 1)$ versus $N_{r p}$ colored by $m_{s}$ as in a). 


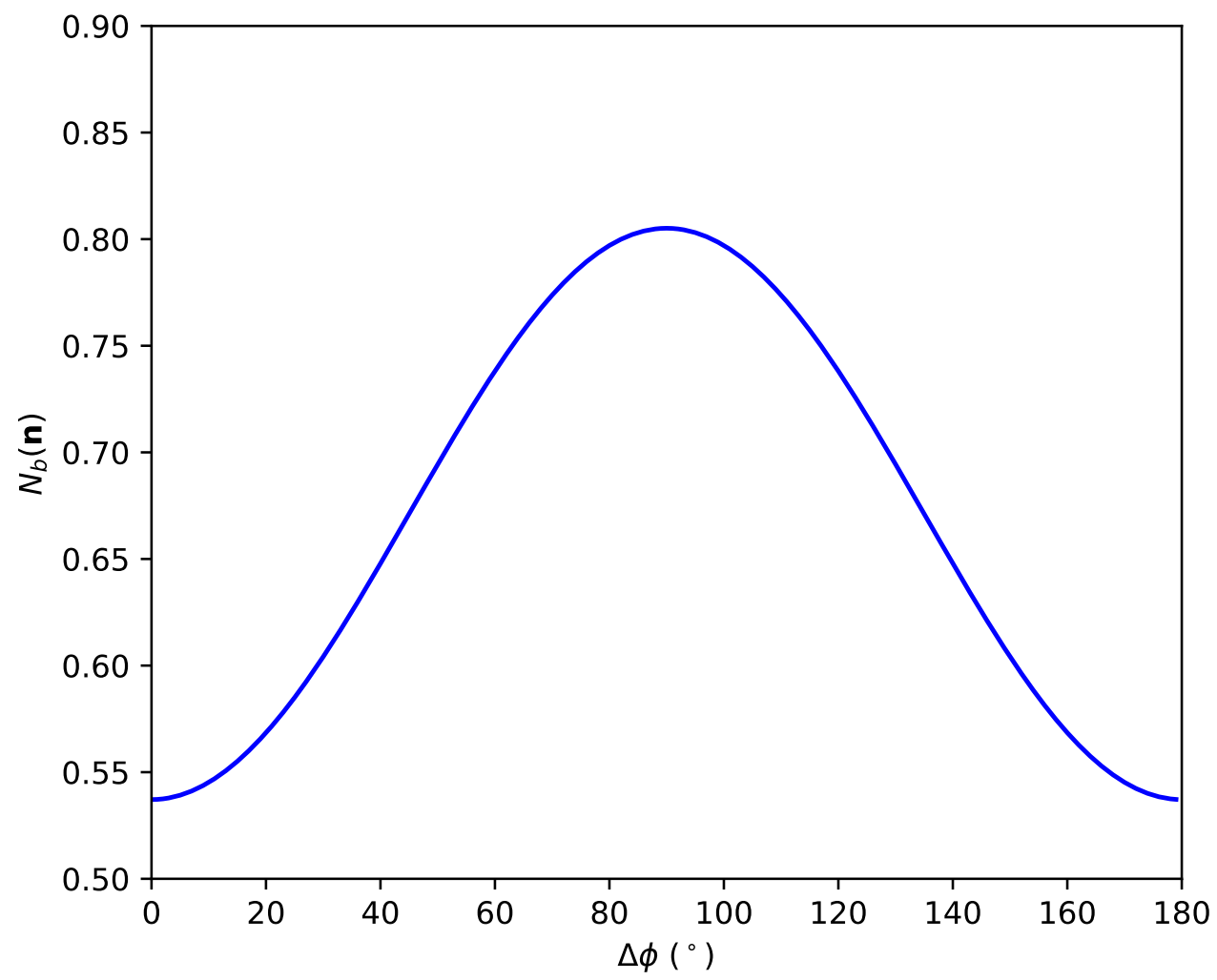

Figure S3. $N_{b}$ values when $\mathbf{n}$ is rotated counterclockwise by $\Delta \phi$ degrees relative to the direction corresponding to $\sigma_{p 1}\left(\mathbf{n}_{p 1}\right)$. 

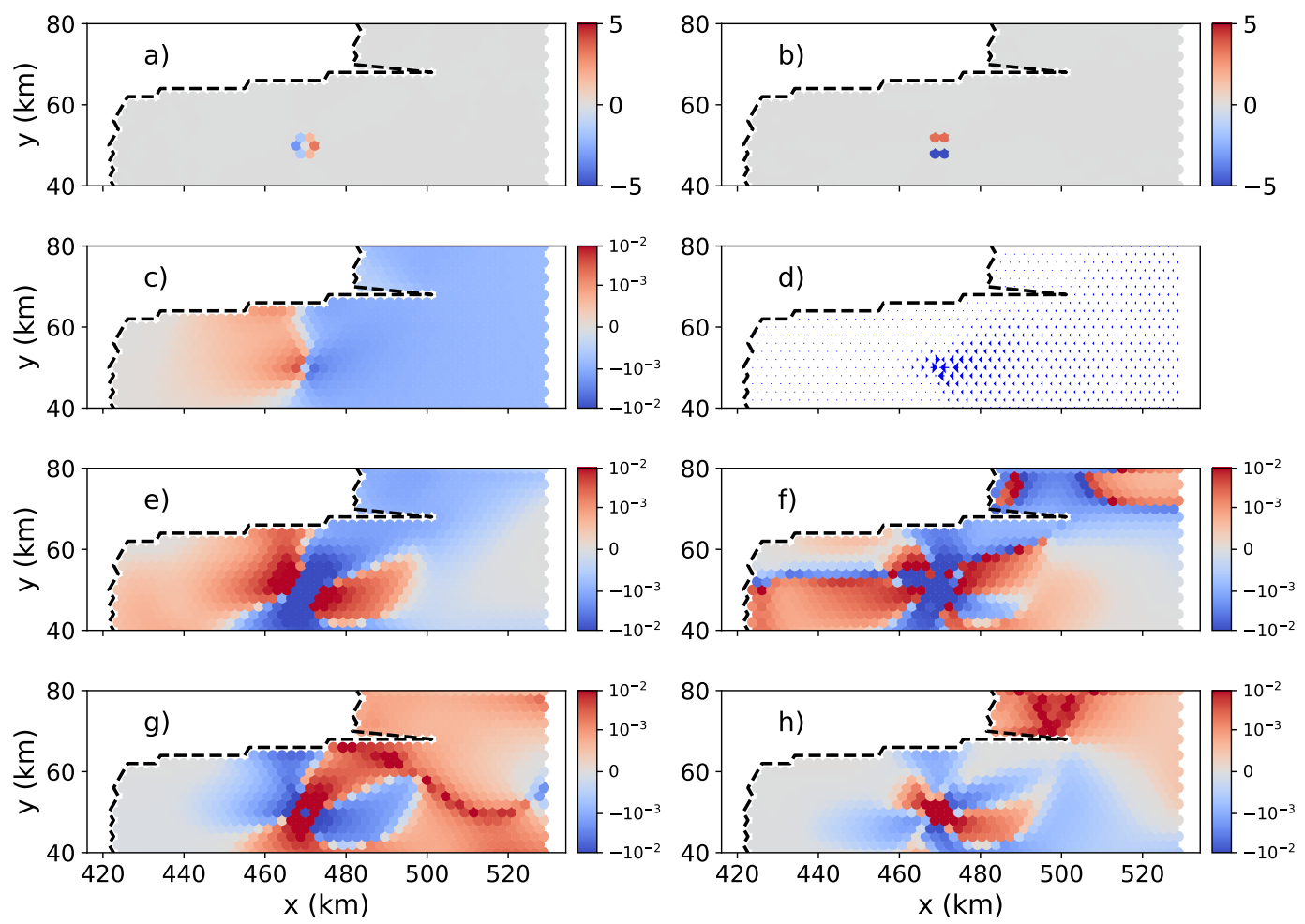

Figure S4. An example of the local change (ratio, in \%) in the ice thickness gradient in $x$ (a), ice thickness gradient in $y$ (b), ice speed (c), ice velocity (d), principal strain rates (e, f), and buttressing number $(\mathrm{g}, \mathrm{h})$ following a local perturbation to the ice shelf thickness. In e) and $\mathrm{g}$ ), changes (colors) are associated with the $\mathbf{n}_{p 1}$ direction and for f) and h) changes are associated with the $\mathbf{n}_{p 2}$ direction. 

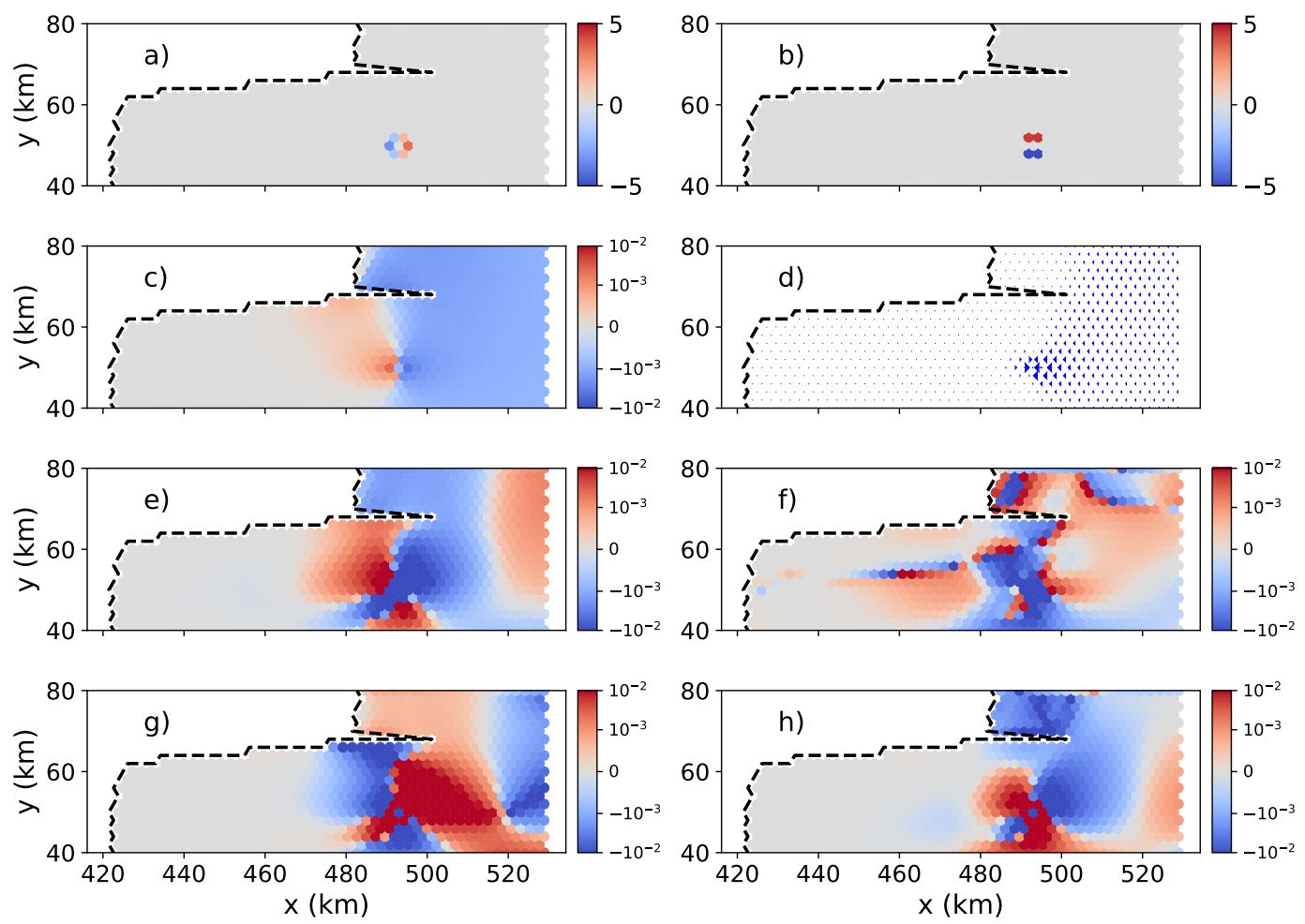

Figure S5. An example of the local change (ratio, in \%) in the ice thickness gradient in $x$ (a), ice thickness gradient in $y$ (b), ice speed (c), ice velocity (d), principal strain rates (e, f), and buttressing number $(\mathrm{g}, \mathrm{h})$ following a local perturbation to the ice shelf thickness. In e) and $\mathrm{g}$ ), changes (colors) are associated with the $\mathbf{n}_{p 1}$ direction and for f) and h) changes are associated with the $\mathbf{n}_{p 2}$ direction. 

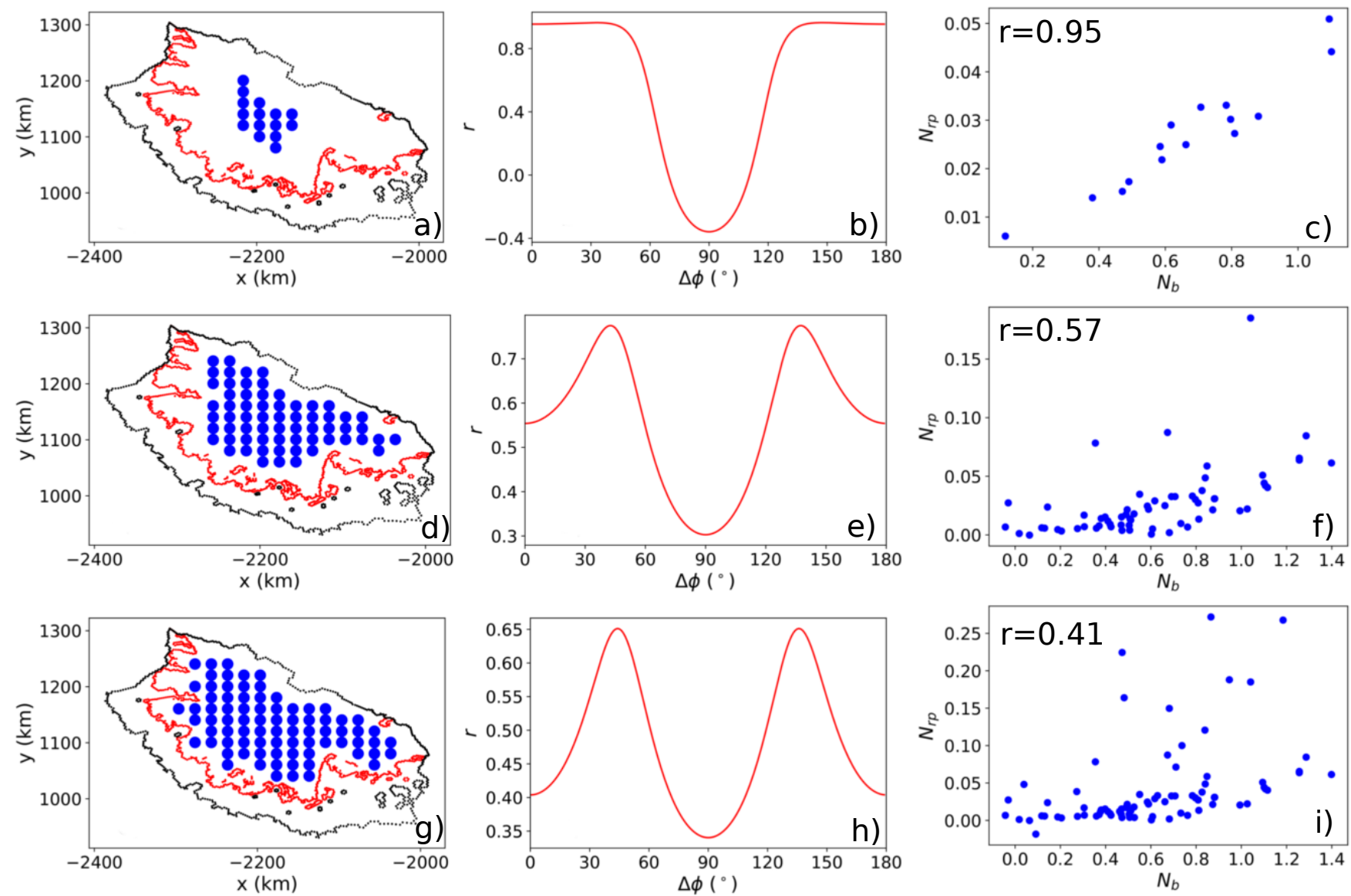

Figure S6. (a, d, g) The locations of the $20 \mathrm{~km} \times 20 \mathrm{~km}$ perturbation boxes (solid blue dots). The red and black dotted lines represent the grounding line and the boundary of model domain, respectively. (b, e, h) The $N_{r p}: N_{b}$ correlation coefficients for each direction rotated counterclockwise from the direction of $\mathbf{n}_{p 1} \cdot(\mathrm{c}, \mathrm{f}, \mathrm{i})$ The corresponding scatter plots for $N_{b}\left(\mathbf{n}_{\mathbf{p} \mathbf{1}}\right)$ and $N_{r p}$. 


\section{References}

30 MacAyeal, D. R.: Ice-shelf backpressure: form drag versus dynamic drag, in: Dynamics of the West Antarctic Ice Sheet, edited by Van Der Veen, C. J. and Oerlemans, J., p. 141, D. Reidel Publishing Company, Dordrecht, Holland, 1987.

Thomas, R. H.: The dynamics of marine ice sheets, The Journal of Glaciology, 24, 167-178, 1979.

Thomas, R. H. and MacAyeal, D. R.: Derived Characteristics of the Ross Ice Shelf, Antarctica, Journal of Glaciology, 28, 397-412, https://doi.org/10.3189/s0022143000005025, 1982. 